\title{
Effect of the Proximal Anastomosis Configuration of the Radial Artery in Patients Undergoing Coronary Artery Bypass Grafting
}

\author{
Seung Keun Yoon, M.D., Hyun Song, M.D., Ph.D., Ju Yong Lim, M.D., Ph.D. \\ Department of Thoracic and Cardiovascular Surgery, Seoul St. Mary's Hospital, College of Medicine, The Catholic University of Korea, Seoul, Korea
}

\section{ARTICLE INFO}

Received June 24, 2020

Revised November 17, 2020

Accepted November 20, 2020

Corresponding author

Ju Yong Lim

Tel $82-2-2258-6769$

Fax 82-2-594-8644

E-mail millalim92@gmail.com

ORCID

https://orcid.org/0000-0003-4961-4890
Background: Several factors, such as the degree of target vessel stenosis, are known to be associated with radial artery (RA) graft patency in coronary artery bypass grafting $(C A B G)$. There is a lack of data regarding the effect of the RA proximal configuration (aortic anastomosis versus T-anastomosis). This study evaluated the effects of the RA proximal configuration on the patency rate and clinical outcomes after CABG.

Methods: We conducted a retrospective study, analyzing 328 patients who had undergone CABG with an RA graft. We divided the patients into 2 groups. The primary endpoint was RA patency and the secondary endpoints were overall mortality and major adverse cardiac and cerebrovascular events (MACCE). We performed a propensity score-matched comparison.

Results: Aorta-RA anastomosis was performed in 275 patients, whereas the rest of the 53 patients received T-RA anastomosis. The mean age was $67.3 \pm 8.7$ years in the T-RA anastomosis group and $63.8 \pm 9.5$ years in the aorta-RA anastomosis group $(p=0.02)$. The mean follow-up duration was $5.13 \pm 3.07$ years. Target vessel stenosis $\geq 70 \%$ (hazard ratio [HR], $0.42 ; 95 \%$ confidence interval [Cl], 0.20-0.91; $\mathrm{p}=0.03$ ) and T-RA anastomosis configuration (HR, 2.34; 95\% Cl, 1.01-5.19; $p=0.04)$ were significantly associated with RA occlusion in the multivariable analysis. However, T-RA anastomosis was not associated with higher risks of overall mortality and MACCE following CABG ( $p=0.30$ and $p=0.07$ in the matched group, respectively).

Conclusion: Aorta-RA anastomosis showed a superior patency rate compared to T-RA anastomosis. However, the RA proximal anastomosis configuration was not associated with mortality or MACCE.

Keywords: Coronary artery bypass grafting, Radial artery, Vascular patency, Vascular graft occlusion, Surgical anastomosis

\section{Introduction}

Coronary artery bypass grafting (CABG) is the treatment of choice for patients with multi-vessel coronary artery disease or left main coronary artery stenosis [1]. Studies have been conducted over recent decades to improve the patency of bypass grafts and to identify the preferred conduit selection, since long-term outcomes are mainly determined by the patency of the bypass grafts used during surgery [2-5]. Using the internal mammary artery (IMA) is known to be the gold standard for conduit selection, and it is highly recommended for grafting the left anterior descending artery (LAD) [6]. However, the selection of the second graft is still controversial. As a second graft, many studies demonstrated superior patency rates of radial artery (RA) grafts compared to saphenous vein grafts (SVGs) [2,7-9]. Deb et al. [8] showed that the occlusion rate of the RA was $8.9 \%(n=269)$, whereas that of the saphenous vein was $18.6 \%(n=269)$ over $7.7 \pm 1.5$ years. Factors such as age, sex, target vessel stenosis, target vessel territory, and graft proximal configuration were suggested as risk factors for RA occlusion [10-17]. Among these factors, the configuration of the proximal anastomosis of RA (direct aorta-RA anastomosis or T-RA anastomosis) remains debatable [1014,18] and only limited data are available. Therefore, we conducted this study to identify the effects of the RA prox- 
imal configuration on the patency rate and clinical outcomes after CABG.

\section{Methods}

\section{Patients}

From January 2010 to August 2018, a total of 874 patients underwent CABG at Seoul St. Mary's Hospital, Seoul, Republic of Korea. We retrospectively reviewed 328 consecutive patients with an RA conduit and excluded patients who were unsuitable for RA grafting due to a positive modified Allen's test, advanced chronic kidney disease ( $\geq$ stage 4 ), or a history of forearm ischemia/trauma (Fig. 1). The data were collected from our thoracic and cardiovascular surgery database and electronic medical records. Follow-up completeness was $97.3 \%$, with a median follow-up duration of 60.25 months (interquartile range [IQR], 28.96-98.43 months). We excluded 34 of the 328 patients from the analysis of RA patency because no follow-up examinations had been performed to confirm patency postoperatively. As the primary interest of our study was to compare the clinical outcomes according to the type of proximal anastomosis of the RA, we divided patients into 2 groups: the aorta-RA anastomosis group and the T-RA anastomosis group. The primary endpoint was RA patency after CABG. Secondary endpoints included overall mortality and major adverse cardiac and cerebrovascular events
(MACCE) (defined as death, myocardial infarction, cerebrovascular accident [CVA], or target vessel revascularization). The institutional review board of the Catholic University College of Medicine approved the study protocol (KC20RASI0500), and the requirement for individual patient consent was waived.

\section{Surgical strategy}

At our institution, we primarily perform on-pump CABG if computed tomography (CT) findings do not rule out cannulation and cross-clamping of the aorta. We considered either on-pump CABG or off-pump CABG, depending on the degree of calcification of the ascending aorta. The left IMA (LIMA) was the first choice of conduit to revascularize the LAD territory, except in contraindicated circumstances. For non-LAD territory, we selected the RA over the SVG, especially for left circumflex territory.

We harvested the RA in a pedicle method, using a Harmonic scalpel (Ethicon Endo Surgery, Cincinnati, OH, USA) to minimize arterial wall damage. After harvesting, we stored the RA in papaverine solution until anastomosis. During the operative and perioperative periods, we administered an intravenous calcium channel blocker (CCB) and nitroglycerine to prevent vasospasms and to induce vasodilation. We then switched to oral CCB and isosorbide dinitrate when oral intake became possible. These medications were continued for more than 1 year postoperatively.

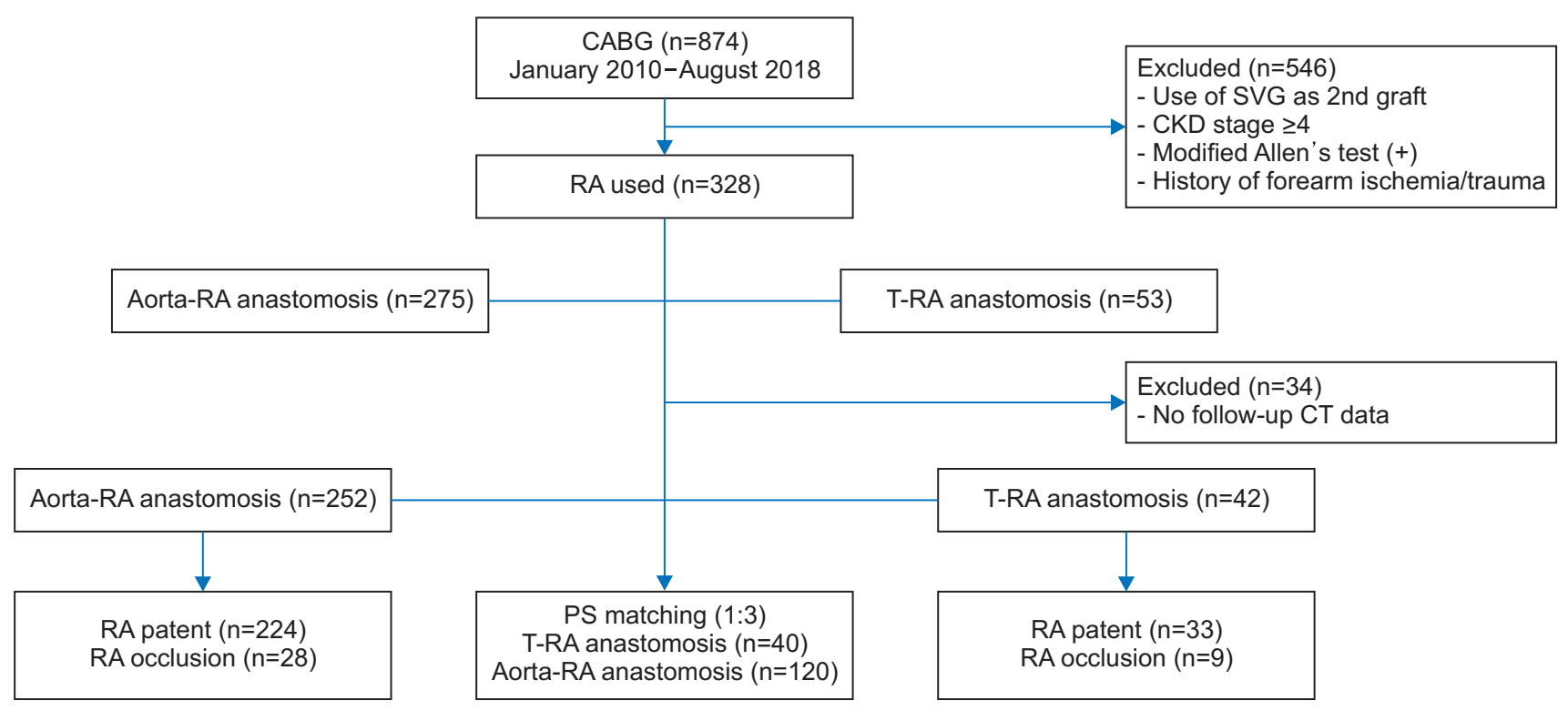

Fig. 1. Flow chart of patient selection. CABG, coronary artery bypass grafting; CKD, chronic kidney disease; CT, computed tomography; PS, propensity score; RA, radial artery; SVG, saphenous vein graft. 
We anastomosed the RA proximally to either the LIMA or SVG as a composite T-graft, or to the ascending aorta. We used continuous 7-0 polypropylene sutures to perform the anastomosis between the aorta and the RA, and continuous 8-0 polypropylene sutures to perform the distal and the T-RA anastomoses. Generally, we preferred aorta-RA anastomoses unless the RA graft was of insufficient length or if there was severe calcification around the aortic anastomosis site.

\section{Follow-up and definition of radial artery patency}

Data collection was performed until February 2020 via direct telephone interviews and a detailed review of all medical records. The date of death in mortality cases were confirmed using information from the National Health Insurance Service.

For the assessment of graft patency, we performed postoperative CT before discharge, 1 year following surgery, and then, every 2 years following surgery. In patients who had poor kidney function (glomerular filtration rate $<50$ $\mathrm{mL} / \mathrm{min} / 1.73 \mathrm{~m}^{2}$ ), we did not perform follow-up CT. We performed coronary angiography in patients who had significant stenotic findings on CT, new-onset angina, or abnormal findings on cardiac echocardiography during follow-up. We based our definition of occlusion on the Fitzgibbon classification, and we considered grade $\mathrm{O}$ to indicate graft occlusion [19]. The degree of target vessel stenosis in patients undergoing sequential anastomosis was defined by a higher degree of stenosis on angiographic findings between the 2 target vessels.

\section{Statistical analysis}

We performed all statistical analyses using IBM SPSS ver. 24.0 (IBM Corp., Armonk, NY, USA). We summarized all data as the mean \pm standard deviation or as the median and IQR for continuous variables and the frequency and the percentage of categorical variables as appropriate. We compared the clinical characteristics between the aorta-RA anastomosis and T-RA anastomosis groups, using the Student $t$-test for the mean of normally distributed continuous variables and the Mann-Whitney U-test for non-parametric skewed distributions. All categorical variables were compared between the 2 groups using the chi-square test or Fisher exact test. To identify risk factors for RA occlusion, mortality, and MACCE, we performed univariable and multivariable analyses using Cox proportional hazard models. The proportional hazards assumption was tested using log-minus-log curves. We found that the assumption of proportional hazards was not violated.

For the multivariable analyses, the variables assessed in univariable models were included in the final multivariable analyses based on their clinical importance if they had a $\mathrm{p}$-value $<0.1$ in the univariable analysis.

To reduce the effect of treatment-selection bias and potential confounders in this observational study, we performed a rigorous adjustment for significant differences in the baseline patient characteristics using propensity score matching. A propensity score was generated for each patient from a multivariable logistic regression model based on 19 preoperative characteristics as independent variables (Tables 1,2). We assessed the discrimination and calibration abilities of the model using the $\mathrm{C}$ and Hosmer-Lemeshow statistics. We matched the T-RA anastomosis group with the aorta-RA anastomosis group in a 1:3 (maximum) ratio using a greedy matching algorithm (the nearestneighbor matching method). The risk of each outcome was compared using a Cox regression model with generalized estimating equations in the propensity score-matched cohort.

Using the Kaplan-Meier method, we assessed freedom from RA occlusion for each anastomosis configuration. We performed inter-group comparisons using the log-rank test and Prentice-Wilcoxon test as appropriate.

\section{Results}

\section{Patient characteristics}

Among the 328 patients, aorta-RA anastomosis was performed in 275 patients, whereas the rest of the 53 patients received T-RA anastomosis. The baseline characteristics of the patients were summarized in Tables 1 and 2 . The mean age was $67.3 \pm 8.7$ years in the T-RA anastomosis group and $63.8 \pm 9.5$ years in the aorta- $R A$ anastomosis group $(\mathrm{p}=0.02)$. The T-RA anastomosis group had a higher proportion of patients with dyslipidemia (30.2\% versus $18.5 \%, \mathrm{p}=0.05$ ) and a longer cardiopulmonary bypass time $(95.67 \pm 58.50$ versus $77.09 \pm 25.74$ minutes, $\mathrm{p}=0.03$ ).

\section{Postoperative results}

In the unmatched population, the T-RA anastomosis group had a statistically significantly higher rate of re-operation due to bleeding $(\mathrm{p}=0.03)$ The overall RA patency rate was $87.4 \%(\mathrm{n}=294)$ during the median follow-up duration of CT scans of 14.73 months (IQR, 5.3-55.1 months). The T-RA anastomosis group showed a higher RA occlu- 
Table 1. General preoperative patient characteristics (unmatched)

\begin{tabular}{|c|c|c|c|}
\hline Characteristic & T-RA anastomosis $(n=53)$ & Aorta-RA anastomosis $(\mathrm{n}=275)$ & p-value \\
\hline Age (yr) & $67.3 \pm 8.7$ & $63.8 \pm 9.5$ & 0.02 \\
\hline Sex (male) & $35(66.0)$ & $195(70.9)$ & 0.48 \\
\hline Diagnosis & & & 0.03 \\
\hline Stable angina & $38(71.7)$ & $128(46.5)$ & \\
\hline Unstable angina & $9(17.0)$ & $98(35.6)$ & \\
\hline Acute myocardial infarction & $6(11.3)$ & $49(17.8)$ & \\
\hline Preoperative ejection fraction (\%) & $53.7 \pm 12.3$ & $56.0 \pm 11.8$ & 0.20 \\
\hline Three-vessel disease & $45(84.9)$ & $211(76.7)$ & 0.13 \\
\hline \multicolumn{4}{|l|}{ Comorbidity } \\
\hline Hypertension & $25(47.2)$ & $174(63.3)$ & 0.03 \\
\hline Diabetes mellitus & $14(26.4)$ & $108(39.3)$ & 0.08 \\
\hline Dyslipidemia & $16(30.2)$ & $51(18.5)$ & 0.05 \\
\hline Current smoker & $8(15.1)$ & $54(19.6)$ & 0.44 \\
\hline Cerebrovascular accident & $6(11.3)$ & $26(9.5)$ & 0.68 \\
\hline Chronic kidney disease (stage 2 or 3 ) & $39(73.6)$ & $186(67.6)$ & 0.39 \\
\hline Atrial fibrillation & $2(3.8)$ & $10(3.6)$ & 0.96 \\
\hline Target vessel stenosis (\%) & & & 0.98 \\
\hline$<70$ & $7(13.2)$ & $36(13.1)$ & \\
\hline$\geq 70$ & $46(86.8)$ & $239(86.9)$ & \\
\hline \multicolumn{4}{|l|}{ Laboratory findings } \\
\hline Preoperative creatinine (mg/dL) & $0.95 \pm 0.28$ & $0.93 \pm 0.27$ & 0.58 \\
\hline Preoperative hemoglobin (g/dL) & $12.80 \pm 1.86$ & $12.96 \pm 1.81$ & 0.56 \\
\hline Preoperative C-reactive protein $(\mathrm{mg} / \mathrm{L})$ & $0.60 \pm 1.20$ & $0.70 \pm 1.50$ & 0.66 \\
\hline
\end{tabular}

Values are presented as mean \pm standard deviation or number (\%).

$\mathrm{RA}$, radial artery.

Table 2. General patient characteristics: operative profiles (unmatched)

\begin{tabular}{|c|c|c|c|}
\hline Characteristic & T-RA anastomosis $(n=53)$ & Aorta-RA anastomosis $(\mathrm{n}=275)$ & p-value \\
\hline Emergency & $1(1.9)$ & $21(7.6)$ & 0.13 \\
\hline Cardiopulmonary bypass strategy & & & $<0.001$ \\
\hline On-pump & $38(71.7)$ & $269(97.8)$ & \\
\hline On-pump, beating & $14(26.4)$ & $3(1.1)$ & \\
\hline Off-pump & $1(1.9)$ & $3(1.1)$ & \\
\hline No. of distal anastomoses & $2.7 \pm 0.7$ & $2.79 \pm 0.69$ & 0.61 \\
\hline Cardiopulmonary bypass time & $95.7 \pm 58.5$ & $77.1 \pm 25.7$ & 0.03 \\
\hline Target vessel stenosis (\%) & $85.0 \pm 16.6$ & $83.5 \pm 15.8$ & 0.52 \\
\hline Total arterial revascularization & $17(32.1)$ & $104(37.8)$ & 0.53 \\
\hline Target vessel diameter (mm) & $1.5 \pm 0.1$ & $1.5 \pm 0.2$ & 0.28 \\
\hline Distal territory of radial artery & & & 0.37 \\
\hline Left anterior descending artery & $12(20.0)$ & $88(28.5)$ & \\
\hline Left circumflex artery & $40(66.7)$ & $179(57.9)$ & \\
\hline Right coronary artery & $8(13.3)$ & $42(13.6)$ & \\
\hline Sequential RA grafta) & $7(13.2)$ & $34(12.4)$ & 0.87 \\
\hline
\end{tabular}

Values are presented as number (\%) or mean \pm standard deviation.

$\mathrm{RA}$, radial artery.

a) The entire target territory of the sequential graft was included. There was only 1 RA occlusion in the T-RA anastomosis group, and no cases of RA occlusion in the aorta-RA anastomosis group.

sion rate than the aorta-RA anastomosis group (21.4\% versus $11.1 \%, \mathrm{p}=0.06$ ) (Table 3 ). The follow-up rate of $\mathrm{CT}$ scans at each time point was $77.4 \%, 47.7 \%$, and $54.5 \%$ after
1 years, 3 years, and 5 years, respectively. The follow-up duration also demonstrated a difference between the groups $(\mathrm{p}=0.02)$. There was no difference between the 2 
Table 3. General postoperative patient characteristics (unmatched)

\begin{tabular}{|c|c|c|c|}
\hline Characteristic & T-RA anastomosis $(n=53)$ & Aorta-RA anastomosis $(\mathrm{n}=275)$ & p-value \\
\hline \multicolumn{4}{|l|}{ Early outcomes ${ }^{\text {a) }}$} \\
\hline In-hospital mortality & $2(3.8)$ & $5(5.9)$ & 0.37 \\
\hline Re-exploration for bleeding & $6(15.1)$ & $11(4.0)$ & 0.03 \\
\hline Wound problem & $5(9.4)$ & $23(8.4)$ & 0.80 \\
\hline Postoperative arrhythmia & $5(9.4)$ & $25(9.1)$ & 0.94 \\
\hline Renal replacement therapy & $3(5.7)$ & $9(3.3)$ & 0.40 \\
\hline Stroke & $1(1.9)$ & $4(1.5)$ & 0.16 \\
\hline \multicolumn{4}{|l|}{ Late outcomes ${ }^{\text {a) }}$} \\
\hline Stroke & 0 & $3(1.79)$ & 0.59 \\
\hline Target vessel revascularization & $1(3.62)$ & $1(0.60)$ & 0.30 \\
\hline Death from any cause & $11(39.60)$ & $38(22.56)$ & 0.20 \\
\hline Myocardial infarction & $1(3.62)$ & $1(0.60)$ & 0.30 \\
\hline Radial artery graft occlusion ${ }^{\text {b) }}$ & $9(45.65)$ & 28 (18.99) & 0.06 \\
\hline Left anterior descending artery & $1(11.1)$ & $8(28.6)$ & \\
\hline Left circumflex artery & $5(55.6)$ & $13(46.4)$ & \\
\hline Right coronary artery & $3(33.3)$ & $7(25.0)$ & \\
\hline LIMA graft occlusionc) & $4(22.43)$ & $11(7.35)$ & 0.11 \\
\hline Follow-up duration (day) & $679.17 \pm 778.75$ & $1,005.39 \pm 1,001.91$ & 0.02 \\
\hline
\end{tabular}

Values are presented as number (\%) or mean \pm standard deviation.

LIMA, left internal mammary artery; RA, radial artery.

${ }^{a}$ Early outcomes: number of events (\%), late outcomes: number of events (/1,000 patient-years). ${ }^{b}$ The analysis was performed on 294 patients who underwent follow-up computed tomography or coronary angiography (T-RA anastomosis: $n=42$, aorta-RA anastomosis: $n=252$ ). ${ }^{c}$ The analysis was performed on 283 patients who underwent coronary artery bypass grafting with LIMA and RA grafts (T-RA anastomosis: $n=37$, aorta-RA anastomosis: $\mathrm{n}=246$ ).

groups in stroke or all-cause mortality $(\mathrm{p}=0.59$ and $\mathrm{p}=0.20$, respectively). We matched 40 T-RA anastomosis patients with 120 aorta-RA anastomosis patients at a 1:3 ratio (Supplementary Table 1).

\section{Risk factor analysis for radial artery occlusion}

In the unmatched population, the univariable analysis showed that age (hazard ratio [HR], 1.04; 95\% confidence interval [CI], 1.00-1.08; $\mathrm{p}=0.04$ ) and the T-RA anastomosis configuration (HR, 3.02; 95\% CI, 1.39-6.58; $\mathrm{p}=0.005$ ) were risk factors for RA occlusion. Target vessel stenosis $\geq 70 \%$ was also related to RA patency (HR, 0.40; 95\% CI, 0.19$0.86 ; \mathrm{p}=0.02$ ) [20]. The multivariable analysis demonstrated that target vessel stenosis $\geq 70 \%$ (HR, $0.42 ; 95 \% \mathrm{CI}$, $0.20-0.91 ; \mathrm{p}=0.03)$ and the T-RA anastomosis configuration (HR, 2.34; 95\% CI, 1.01-5.19; $\mathrm{p}=0.04)$ were significant predictors of RA occlusion (Table 4).

Freedom from RA occlusion was found in $91.3 \%, 88.5 \%$, and $82.8 \%$ of patients after 2 years, 4 years, and 6 years, respectively, in the aorta-RA group, whereas freedom from RA occlusion was observed in $85.4 \%, 63.2 \%$, and $37.9 \%$, respectively, in the T-RA group ( $\mathrm{p}=0.002$ ) (Fig. $2 \mathrm{~A}$ ). In the matched cohort, freedom from RA occlusion was higher in aorta-RA group, as shown in Fig. 2B ( $\mathrm{p}=0.003)$.

\section{Risk factor analysis for overall mortality}

The configuration of the proximal anastomosis was not implicated in overall mortality ( $\mathrm{p}=0.11$ ) (Table 5). The multivariable analysis showed that preoperative ejection fraction (HR, 0.97; 95\% CI, 0.95-0.99; $\mathrm{p}=0.002$ ), preoperative hemoglobin (HR, 0.77; 95\% CI, 0.67-0.89; $<<0.001)$ and preoperative C-reactive protein levels (HR, 1.20; 95\% CI, 1.061.36; $\mathrm{p}=0.01$ ) were related to overall mortality. There were no significant differences between groups in terms of freedom from mortality in the matched patients $(\mathrm{p}=0.50)$ (Fig. 3A).

\section{Risk factor analysis for major adverse cardiac and cerebrovascular events}

The configuration of RA was marginally statistically associated with MACCE $(\mathrm{p}=0.06)$ in the univariable analysis. However, the multivariable analysis showed no significant associations $(\mathrm{p}=0.17)$. Preoperative ejection fraction was linked to MACCE (HR, 0.97; 95\% CI, 0.95-0.99; $\mathrm{p}=0.001$ ). Preoperative hemoglobin was also an important factor associated with MACCE (HR, 0.76; 95\% CI, 0.67-0.87; $\mathrm{p}<0.001$ ) (Table 6). There were no significant findings between groups concerning freedom from MACCE in the matched patients ( $\mathrm{p}=0.16$ ) (Fig. 3B). 
Table 4. Risk factors for occlusion of the radial artery graft in unmatched patients

\begin{tabular}{|c|c|c|c|c|}
\hline \multirow{2}{*}{ Variable } & \multicolumn{2}{|c|}{ Univariable } & \multicolumn{2}{|c|}{ Multivariable } \\
\hline & $\operatorname{HR}(95 \% \mathrm{Cl})$ & p-value & $\mathrm{HR}(95 \% \mathrm{Cl})$ & p-value \\
\hline Age $(y r)$ & $1.04(1.00-1.08)$ & 0.04 & $1.04(0.99-1.08)$ & 0.10 \\
\hline Female & $1.11(0.56-2.19)$ & 0.77 & & \\
\hline \multicolumn{5}{|l|}{ Diagnosis } \\
\hline Stable angina & 1 & & & \\
\hline Unstable angina & $0.68(0.25-1.83)$ & 0.44 & & \\
\hline Acute myocardial infarction & $1.10(0.53-2.26)$ & 0.80 & & \\
\hline Preoperative ejection fraction (\%) & $1.01(0.98-1.04)$ & 0.58 & & \\
\hline Hypertension & $1.28(0.64-2.56)$ & 0.49 & & \\
\hline Diabetes mellitus & $1.89(0.98-3.63)$ & 0.06 & & \\
\hline Dyslipidemia & $1.85(0.87-3.97)$ & 0.11 & & \\
\hline Current smoker & $1.08(0.47-2.46)$ & 0.86 & & \\
\hline Chronic kidney disease & $2.50(1.09-5.71)$ & 0.03 & & \\
\hline Cerebrovascular accident & $1.06(0.37-3.01)$ & 0.91 & & \\
\hline Preoperative creatinine (mg/dL) & $1.80(0.70-4.64)$ & 0.22 & & \\
\hline Preoperative hemoglobin (g/dL) & $0.86(0.72-1.03)$ & 0.10 & & \\
\hline Preoperative C-reactive protein (mg/L) & $0.86(0.65-1.23)$ & 0.49 & & \\
\hline \multicolumn{5}{|l|}{ Target vessel stenosis (\%) } \\
\hline$<70$ & 1 & & 1 & \\
\hline$\geq 70$ & $0.40(0.19-0.86)$ & 0.02 & $0.42(0.20-0.91)$ & 0.03 \\
\hline Emergency & $0.04(0.00-11.18)$ & 0.27 & & \\
\hline Cardiopulmonary bypass time (min) & $1.00(0.98-1.01)$ & 0.61 & & \\
\hline \multicolumn{5}{|l|}{ Proximal anastomosis } \\
\hline Aorta (direct) & 1 & & 1 & \\
\hline T-graft group & $3.02(1.39-6.58)$ & 0.005 & $2.34(1.01-5.19)$ & 0.04 \\
\hline \multicolumn{5}{|l|}{ Distal area } \\
\hline Left anterior descending artery & 1 & & & \\
\hline Left circumflex artery & $0.86(0.38-1.94)$ & 0.71 & & \\
\hline Right coronary artery & $1.19(0.470-3.00)$ & 0.72 & & \\
\hline Sequential radial artery & $0.26(0.036-1.93)$ & 0.19 & & \\
\hline
\end{tabular}

$\mathrm{Cl}$, confidence interval; $\mathrm{HR}$, hazard ratio.

A

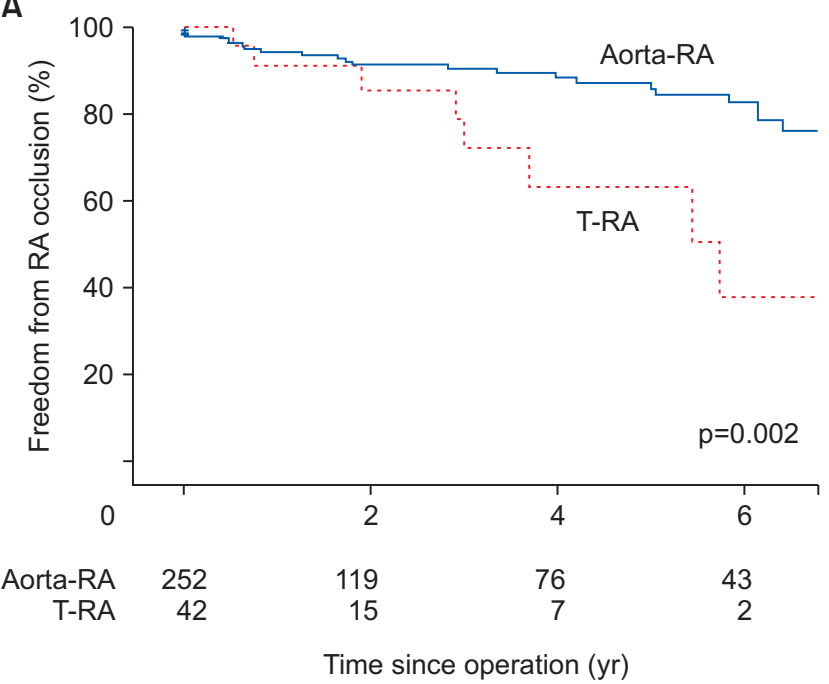

B

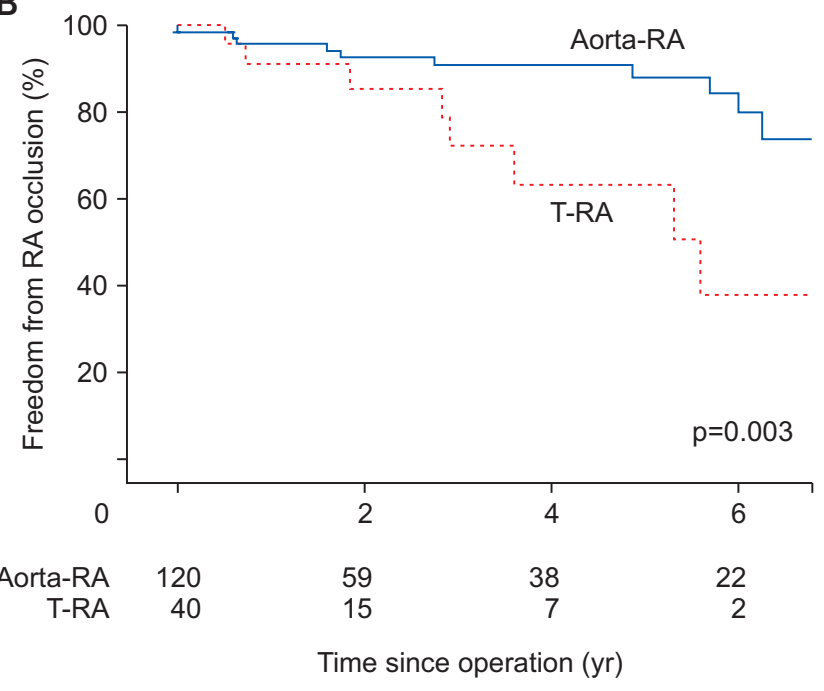

Fig. 2. (A) Freedom from RA occlusion in the aorta-RA anastomosis group and T-RA anastomosis group in the unmatched cohort. (B) Results for the matched cohort. RA, radial artery. 
Table 5. Risk factors for overall mortality in unmatched patients

\begin{tabular}{|c|c|c|c|c|}
\hline \multirow{2}{*}{ Variable } & \multicolumn{2}{|c|}{ Univariable } & \multicolumn{2}{|c|}{ Multivariable } \\
\hline & $\mathrm{HR}(95 \% \mathrm{Cl})$ & p-value & $\mathrm{HR}(95 \% \mathrm{Cl})$ & p-value \\
\hline Age (yr) & $1.04(1.01-1.08)$ & 0.02 & & \\
\hline Female & $1.02(0.56-1.85)$ & 0.95 & & \\
\hline Preoperative ejection fraction (\%) & $0.96(0.95-0.98)$ & $<0.001$ & $0.97(0.95-0.99)$ & 0.002 \\
\hline Hypertension & $0.72(0.41-1.27)$ & 0.26 & & \\
\hline Diabetes mellitus & $0.75(0.41-1.37)$ & 0.34 & & \\
\hline Dyslipidemia & $0.69(0.31-1.53)$ & 0.36 & & \\
\hline Current smoker & $0.96(0.47-1.96)$ & 0.92 & & \\
\hline Chronic kidney disease & $1.16(0.63-2.13)$ & 0.64 & & \\
\hline Cerebrovascular accident & $1.28(0.46-3.56)$ & 0.64 & & \\
\hline Preoperative creatinine (mg/dL) & $2.56(1.11-5.92)$ & 0.03 & & \\
\hline Preoperative hemoglobin (g/dL) & $0.76(0.66-0.87)$ & $<0.001$ & $0.77(0.67-0.89)$ & $<0.001$ \\
\hline Preoperative C-reactive protein (mg/L) & $1.30(1.16-1.46)$ & $<0.001$ & $1.20(1.06-1.36)$ & 0.01 \\
\hline \multicolumn{5}{|l|}{ Target vessel stenosis $(\%)$} \\
\hline$<70$ & 1 & & 1 & \\
\hline$\geq 70$ & $3.68(0.894-15.16)$ & 0.07 & $3.12(0.76-12.91)$ & 0.12 \\
\hline Emergency operation & $0.91(0.28-2.92)$ & 0.87 & & \\
\hline Cardiopulmonary bypass time (min) & $1.01(1.00-1.01)$ & 0.03 & & \\
\hline \multicolumn{5}{|l|}{ Proximal anastomosis } \\
\hline Aorta (direct) & 1 & & & \\
\hline T-graft group & $1.73(0.88-3.40)$ & 0.11 & & \\
\hline \multicolumn{5}{|l|}{ Distal area } \\
\hline Left anterior descending artery & 1 & & & \\
\hline Left circumflex artery & $1.51(0.67-3.42)$ & 0.33 & & \\
\hline Right coronary artery & $1.67(0.64-4.39)$ & 0.30 & & \\
\hline Sequential radial artery & $1.96(0.95-4.05)$ & 0.07 & & \\
\hline RA patency & $0.76(0.27-2.15)$ & 0.60 & & \\
\hline
\end{tabular}

$\mathrm{Cl}$, confidence interval; HR, hazard ratio.

A

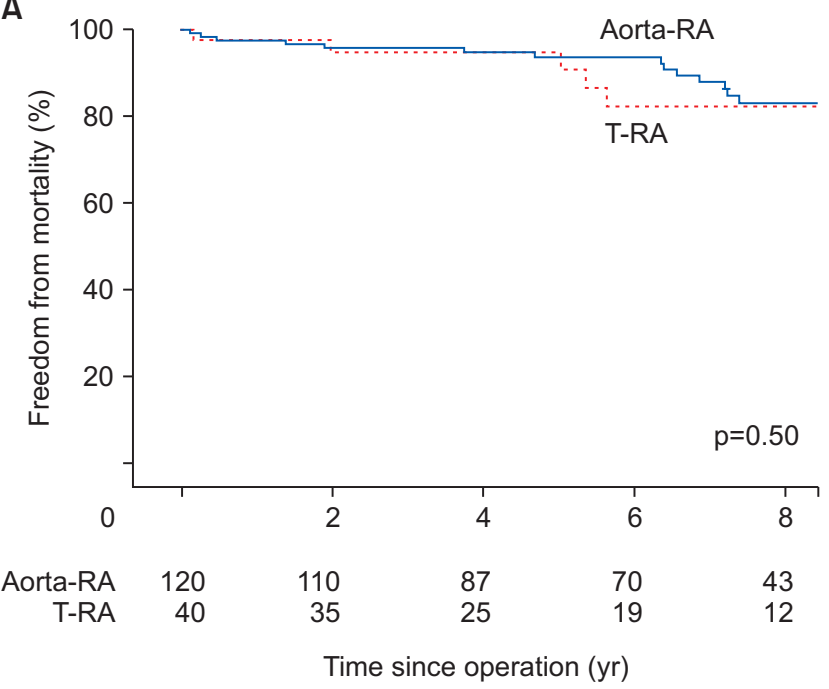

B

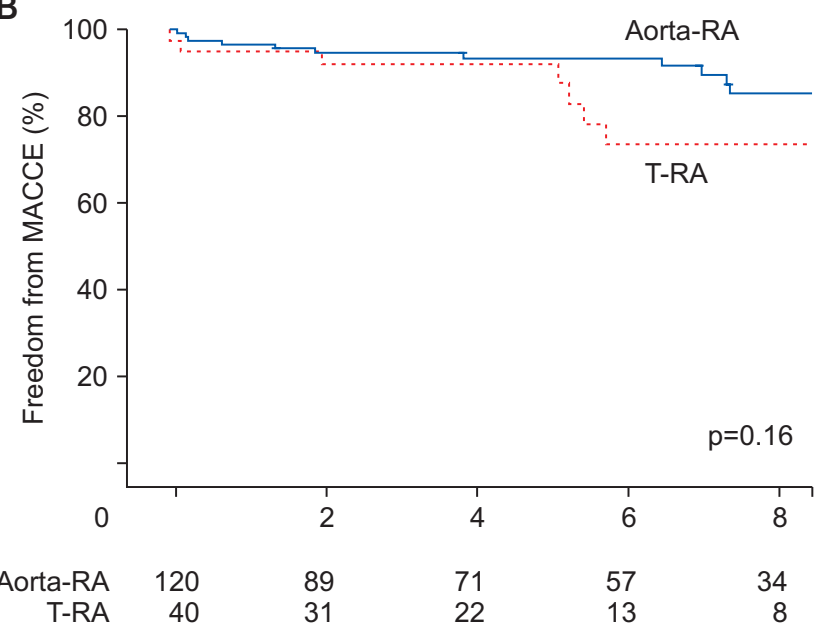

Fig. 3. (A) Freedom from mortality. (B) Freedom from MACCE in the aorta-RA anastomosis group and T-RA anastomosis group in the matched cohort. MACCE, major adverse cardiac and cerebrovascular event; RA, radial artery. 
Table 6. Risk factors for major adverse cardiac and cerebrovascular event in unmatched patients

\begin{tabular}{|c|c|c|c|c|}
\hline \multirow{2}{*}{ Variable } & \multicolumn{2}{|c|}{ Univariable } & \multicolumn{2}{|c|}{ Multivariable } \\
\hline & $\operatorname{HR}(95 \% \mathrm{CI})$ & p-value & $\operatorname{HR}(95 \% \mathrm{Cl})$ & p-value \\
\hline Age (yr) & $1.03(1.01-1.07)$ & 0.02 & & \\
\hline Female & $1.04(0.59-1.83)$ & 0.89 & & \\
\hline Preoperative ejection fraction (\%) & $0.97(0.95-0.98)$ & $<0.001$ & $0.97(0.95-0.99)$ & 0.001 \\
\hline Hypertension & $0.66(0.39-1.12)$ & 0.12 & & \\
\hline Diabetes mellitus & $0.90(0.52-1.57)$ & 0.72 & & \\
\hline Dyslipidemia & $0.59(0.27-1.29)$ & 0.19 & & \\
\hline Current smoker & $0.83(0.40-1.69)$ & 0.60 & & \\
\hline Chronic kidney disease & $1.38(0.76-2.50)$ & 0.29 & & \\
\hline Cerebrovascular accident & $1.13(0.45-2.84)$ & 0.79 & & \\
\hline Preoperative creatinine (mg/dL) & $2.55(1.15-5.63)$ & 0.02 & & \\
\hline Preoperative hemoglobin (g/dL) & $0.76(0.67-0.86)$ & $<0.001$ & $0.76(0.67-0.87)$ & $<0.001$ \\
\hline Preoperative C-reactive protein $(\mathrm{mg} / \mathrm{L})$ & $1.27(1.14-1.43)$ & $<0.001$ & $1.16(1.03-1.32)$ & 0.02 \\
\hline \multicolumn{5}{|l|}{ Target vessel stenosis $(\%)$} \\
\hline$<70$ & 1 & & & \\
\hline$\geq 70$ & $2.68(0.84-8.56)$ & 0.10 & & \\
\hline Emergency operation & $0.79(0.25-2.53)$ & 0.69 & & \\
\hline Cardiopulmonary bypass time (min) & $1.01(0.10-1.01)$ & 0.08 & & \\
\hline \multicolumn{5}{|l|}{ Proximal anastomosis } \\
\hline Aorta (direct) & 1 & & & \\
\hline T-graft group & $1.84(0.99-3.44)$ & 0.06 & & \\
\hline \multicolumn{5}{|l|}{ Distal area } \\
\hline Left anterior descending artery & 1 & & & \\
\hline Left circumflex artery & $1.49(0.69-3.21)$ & 0.31 & & \\
\hline Right coronary artery & $1.62(0.65-4.03)$ & 0.30 & & \\
\hline Radial artery sequential & $2.20(1.13-4.26)$ & 0.02 & & \\
\hline
\end{tabular}

$\mathrm{Cl}$, confidence interval; HR, hazard ratio.

Table 7. Analysis of direct aorta-RA configuration-related outcomes in the propensity-matched cohort

\begin{tabular}{lccc}
\hline \multicolumn{1}{c}{ Outcomes } & No. of events $(\%)$ & OR $(95 \% \mathrm{Cl})$ & $\mathrm{p}$-value \\
\hline RA occlusion & & & 1.00 \\
Aorta-RA anastomosis $)^{\mathrm{a}}$ & $12(10.0)$ & $3.58(1.46-8.77)$ & 0.005 \\
T-RA anastomosis & $9(22.5)$ & 1.00 & 0.30 \\
Overall mortality & $14(11.7)$ & $1.63(0.65-4.07)$ & \\
Aorta-RA anastomosis & $7(17.0)$ & 1.00 & 0.07 \\
T-RA anastomosis & & $2.35(0.98-5.60)$ & \\
MACCE & $12(10.0)$ & $9(22.5)$ & \\
Aorta-RA anastomosis & & \\
T-RA anastomosis &
\end{tabular}

$\mathrm{Cl}$, confidence interval; MACCE, major adverse cardiac and cerebrovascular event; OR, odds ratio; RA, radial artery.

a) The total number of patients in the aorta-RA anastomosis group was $n=120$, and that of the T-RA anastomosis group was $n=40$.

\section{Outcomes in the propensity score-matched cohort Discussion}

T-RA anastomosis configuration was identified as a significant risk factor for RA occlusion (HR, 3.58; 95\% CI, 1.46-8.77; $\mathrm{p}=0.005)$. However, similar to the unmatched data, there was no statistically significant correlation with overall mortality (HR, 1.63; 95\% CI, 0.65-4.07; $\mathrm{p}=0.30$ ) or MACCE (HR, 2.35; 95\% CI, 0.98-5.60; $\mathrm{p}=0.07$ ) (Table 7).
Our study indicated that the aorta-RA anastomosis configuration showed superior patency compared to the T-RA anastomosis configuration. However, this had no statistical significance for overall mortality or MACCE. The risk factors for RA occlusion consisted of target vessel stenosis and proximal anastomosis configuration. Preoperative ejection 
fraction and preoperative hemoglobin levels were significantly associated with mortality and MACCE.

Several previous studies regarding RA proximal configuration and patency reported no significant association. Kim et al. [10] demonstrated that the proximal anastomosis configuration was not significant if the degree of stenosis and distal territory of the target vessel are considered in the off-pump coronary artery bypass (OPCAB) group. In other studies, the proximal anastomosis configuration was found to be unrelated to patency [11-14]. They recommended T-RA anastomosis to minimize aortic manipulation, as well as using a longer length of graft to increase its usefulness. Kim et al. [10] analyzed a comparatively small group of patients who had undergone OPCAB. The study of Lemma et al. [11] also had limitations because the rate of patients who received patency evaluation after surgery was low (aorta-coronary group, 12\%; Y graft group, 25.6\%). The results may differ because of these factors.

Jung et al. [18] reported that the aorta-direct group showed better early and late patency rates than did the T-graft group. Those findings align with our results.

The reasons for these conflicting findings are unclear. There are conflicting theories in relation to the underlying physiology. When aorta-RA anastomosis is performed, intimal hyperplasia may be induced because the intima of the RA receives greater stress than the wall receives in the native position of the RA [17]. However, other studies have considered this to be an advantage in overcoming competing forces [14].

Regarding CVA risk, which is one of the biggest drawbacks of aorta-RA anastomosis, there were no statistically significant differences in the above studies, or in ours $[10,12]$.

This study showed that the degree of target vessel stenosis was related to RA patency. This finding has already been demonstrated in other studies [15,16]; however, we confirmed that the configuration of RA graft was not related to mortality or MACCE. In other words, occlusion of the RA graft did not affect the risk of mortality and MACCE. A reason for this may be the limited clinical significance of the second graft. The preoperative ejection fraction and preoperative hemoglobin were identified as meaningful factors for both long-term outcomes. Preoperative ejection fraction is a well-known risk factor in cardiac surgery, and is used as a factor in the EuroSCORE [21]. The preoperative hemoglobin level is an indicator of the patient's condition before surgery and is predicted to act as a risk factor for long-term outcomes in relation to age and renal function $[22,23]$.
This study has several limitations. First, the data were collected from a single institution. Therefore, the number of patients was relatively small. Additionally, this was a retrospective and non-randomized study. To overcome this limitation, we implemented propensity score matching. Nevertheless, confounding factors could not be ruled out. Second, the time of occlusion was assumed to be the date of postoperative cardiac CT or coronary angiography, although that date is unlikely to reflect the actual date of occlusion development. Third, although some patients did not properly receive CT or coronary angiography evaluation to ascertain patency because of kidney function deterioration or because they were lost to follow-up, the number of such patients was relatively small (10.4\% out of 328$)$. Therefore, the results were not significantly affected. Finally, the T-RA anastomosis group in our study seemed heterogeneous, including T-configurations from 2 different inflows (SVG and LIMA), which could have been a potential source of bias.

In conclusion, aorta-RA anastomosis showed a superior patency rate compared to T-RA anastomosis. More than $70 \%$ target vessel stenosis was associated with favorable patency. The RA proximal configuration was unrelated to mortality or MACCE. Future studies may be necessary to ascertain the relationship between the RA proximal anastomosis configuration and RA patency.

\section{Conflict of interest}

No potential conflict of interest relevant to this article was reported.

\section{ORCID}

Seung Keun Yoon: https://orcid.org/0000-0002-2609-2148 Hyun Song: https://orcid.org/0000-0003-2650-8104

Ju Yong Lim: https://orcid.org/0000-0003-4961-4890

\section{Supplementary materials}

Supplementary materials can be found via https://doi. org/10.5090/jcs.20.082. Supplementary Table 1. Patient characteristics of the propensity score-matched cohort.

\section{References}

1. Epstein AJ, Polsky D, Yang F, Yang L, Groeneveld PW. Coronary revascularization trends in the United States, 2001-2008. JAMA 2011; 305:1769-76. 
2. Carrel T, Winkler B. Current trends in selection of conduits for coronary artery bypass grafting. Gen Thorac Cardiovasc Surg 2017;65: 549-56.

3. Acar C, Jebara VA, Portoghese M, et al. Revival of the radial artery for coronary artery bypass grafting. Ann Thorac Surg 1992;54:65260 .

4. Naito Y, Shinoka T, Duncan D, et al. Vascular tissue engineering: towards the next generation vascular grafts. Adv Drug Deliv Rev 2011; 63:312-23.

5. Loop FD, Lytle BW, Cosgrove DM, et al. Influence of the internal-mammary-artery graft on 10-year survival and other cardiac events. N Engl J Med 1986;314:1-6.

6. Neumann FJ, Sousa-Uva M, Ahlsson A, et al. 2018 ESC/EACTS guidelines on myocardial revascularization. Eur Heart J 2019;40:87165 .

7. Locker C, Schaff HV, Dearani JA, et al. Multiple arterial grafts improve late survival of patients undergoing coronary artery bypass graft surgery: analysis of 8622 patients with multivessel disease. Circulation 2012;126:1023-30.

8. Deb S, Cohen EA, Singh SK, et al. Radial artery and saphenous vein patency more than 5 years after coronary artery bypass surgery: results from RAPS (Radial Artery Patency Study). J Am Coll Cardiol 2012;60:28-35.

9. Gaudino M, Benedetto U, Fremes S, et al. Radial-artery or saphenous-vein grafts in coronary-artery bypass surgery. N Engl J Med 2018;378:2069-77.

10. Kim DJ, Lee SH, Joo HC, Yoo KJ, Youn YN. Effect of the proximal anastomosis site on mid-term radial artery patency in off-pump coronary artery bypass. Eur J Cardiothorac Surg 2018;54:475-82.

11. Lemma M, Mangini A, Gelpi G, Innorta A, Spina A, Antona C. Is it better to use the radial artery as a composite graft?: clinical and angiographic results of aorto-coronary versus Y-graft. Eur J Cardiothorac Surg 2004;26:110-7.

12. Iaco AL, Teodori G, Di Giammarco G, et al. Radial artery for myocardial revascularization: long-term clinical and angiographic results.
Ann Thorac Surg 2001;72:464-9.

13. Maniar HS, Barner HB, Bailey MS, et al. Radial artery patency: are aortocoronary conduits superior to composite grafting? Ann Thorac Surg 2003;76:1498-504.

14. Sajja LR, Mannam G, Pantula NR, Sompalli S. Role of radial artery graft in coronary artery bypass grafting. Ann Thorac Surg 2005;79: 2180-8.

15. Maniar HS, Sundt TM, Barner HB, et al. Effect of target stenosis and location on radial artery graft patency. J Thorac Cardiovasc Surg 2002;123:45-52.

16. Mussa S, Choudhary BP, Taggart DP. Radial artery conduits for coronary artery bypass grafting: current perspective. J Thorac Cardiovasc Surg 2005;129:250-3.

17. Calafiore AM, Teodori G, Di Giammarco G, et al. Coronary revascularization with the radial artery: new interest for an old conduit. J Card Surg 1995;10:140-6.

18. Jung SH, Song H, Choo SJ, et al. Comparison of radial artery patency according to proximal anastomosis site: direct aorta to radial artery anastomosis is superior to radial artery composite grafting. J Thorac Cardiovasc Surg 2009;138:76-83.

19. Fitzgibbon GM, Kafka HP, Leach AJ, Keon WJ, Hooper GD, Burton JR. Coronary bypass graft fate and patient outcome: angiographic follow-up of 5,065 grafts related to survival and reoperation in 1,388 patients during 25 years. J Am Coll Cardiol 1996;28:616-26.

20. Cameron J, Trivedi S, Stafford G, Bett JH. Five-year angiographic patency of radial artery bypass grafts. Circulation 2004;110(11 Suppl 1): II23-6.

21. Nashef SA, Roques F, Sharples LD, et al. EuroSCORE II. Eur J Cardiothorac Surg 2012;41:734-45.

22. Kulier A, Levin J, Moser R, et al. Impact of preoperative anemia on outcome in patients undergoing coronary artery bypass graft surgery. Circulation 2007;116:471-9.

23. LaPar DJ, Hawkins RB, McMurry TL, et al. Preoperative anemia versus blood transfusion: which is the culprit for worse outcomes in cardiac surgery? J Thorac Cardiovasc Surg 2018;156:66-74. 